Research Paper

\title{
Clinicopathological Significance and Antitumor Effect of MPHOSPHI in Testicular Germ Cell Tumor
}

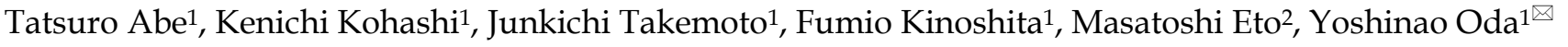 \\ 1. Department of Anatomic Pathology, Graduate School of Medical Sciences, Kyushu University, 3-1-1 Maidashi, Higashi-ku, Fukuoka 812-8582, Japan \\ 2. Department of Urology, Graduate School of Medical Sciences, Kyushu University, 3-1-1 Maidashi, Higashi-ku, Fukuoka 812-8582, Japan \\ $\triangle$ Corresponding author: Yoshinao Oda, Department of Anatomic Pathology, Pathological Sciences, Graduate School of Medical Sciences, Kyushu University, \\ Maidashi 3-1-1, Higashi-ku, Fukuoka 812-8582, Japan. E-mail: oda@surgpath.med.kyushu-u.ac.jp; Tel: +81-92-642-6061; FAX: +81-92-642-5968 \\ (C) Ivyspring International Publisher. This is an open access article distributed under the terms of the Creative Commons Attribution (CC BY-NC) license \\ (https://creativecommons.org/licenses/by-nc/4.0/). See http://ivyspring.com/terms for full terms and conditions.
}

Received: 2018.02.01; Accepted: 2018.07.18; Published: 2018.10.31

\begin{abstract}
MPHOSPHI, which is one of the kinesin superfamily proteins, has been reported to play an essential role in the carcinogenesis and progression of several kinds of cancers. MPHOSPHI has also been suggested to be involved in STAT3 phosphorylation in hepatocellular carcinoma. However, the biological behavior of MPHOSPHI in testicular germ cell tumors (TGCTs) is unclear at present. The purposes of this study were to investigate the correlation between the expression of MPHOSPHI and clinicopathological factors and to examine the efficacy of MPHOSPHI target therapy in TGCTs. We investigated 75 formalin-fixed paraffin-embedded TGCT samples, containing a total of 86 germ cell tumor components, by immunohistochemistry and 12 frozen samples by Western blotting. Moreover, we carried out in vitro studies to clarify the antitumor effect of MPHOSPHI knockdown in embryonal carcinoma cell lines, NEC8 and NEC14, using small interference RNA (siRNA). A significantly high expression of MPHOSPH1 was recognized in embryonal carcinoma and yolk sac tumor components compared to the seminoma component ( $p<0.001$, respectively). Clinically, non-seminoma cases are known to have worse prognosis than pure-seminoma cases. Interestingly, high MPHOSPHI expression was associated with distant metastasis $(p=0.001)$, and thus with advanced-stage disease in this study. High expression of MPHOSPH1 interacted with high expression of phosphorylated STAT3 $(p=0.01)$. The in vitro experiments demonstrated that MPHOSPHI interruption by siRNA resulted in a significant reduction of cell migration, invasion, proliferation and colony formation in both embryonal carcinoma cell lines $(p<0.001$, respectively). In conclusion, MPHOSPHI may be a potential treatment option for TGCTs, and its expression may be a novel biomarker of poor prognosis.
\end{abstract}

Key words: MPHOSPH1; testicular germ cell tumor; seminoma; embryonal carcinoma, yolk sac tumor

\section{Introduction}

Testicular germ cell tumor (TGCT) is a relatively rare disease with an incidence rate is 1.5-10.5 per 100,000 men [1]. It commonly occurs in young men, with peak incidence in the 20 s to 30 s. And in men under 45 years of age, it is the most common malignancy, accounting for $17 \%$ of all cancers in that subgroup [2]. Clinically, non-seminomas such as embryonal carcinoma, yolk sac tumor, choriocarcinoma and teratoma have a worse prognosis than pure seminoma [3]. The survival rate in TGCTs has improved since the early 1980s by the introduction of cisplatin-based chemotherapy, but treatment-resistant cases still exist $[4,5]$.
M-phase phosphoprotein 1 (MPHOSPH1), also referred to as KIF20B, is one of the kinesin superfamily (KIF) proteins [6]. KIF proteins move microtubules toward the plus-end direction with ATPase activity and play an essential role in cytokinesis, including cytokinesis in mitosis or meiosis. MPHOSPH1 has been reported to be the phosphorylated protein at the G2/M transition, and to complete the cytokinesis in the late telophase of mitosis [6, 7]. On the other hand, other articles have reported that MPHOSPH1 is a cancer-testis antigen and potentially an ideal target for cancer immunotherapy $[8,9]$. 
MPHOSPH1 has been shown to be up-regulated in many types of cancer cells $[8,10]$. In keeping with these findings, knockdown of MPHOSPH1 was shown to suppress tumor cell progression in urothelial carcinoma and hepatocellular carcinoma [8, 11]. In hepatocellular carcinoma, MPHOSPH1 knockdown was shown to stabilize p53 and inhibited phosphorylation of STAT3 [11]. Other investigations revealed that SOCS3 was involved in suppressing and thereby inactivating the STAT3 pathway $[12,13]$.

In this study, we examined MPHOSPH1, pSTAT3 and SOCS3 expression in clinical specimens by both immunohistochemistry and Western blotting. We then investigated the clinical and pathological significance of MPHOSPH1 in TGCT patients. Finally, we performed in vitro assays using embryonal carcinoma cell lines, and found that MPHOSPH1 was a potential therapeutic target.

\section{Materials and methods}

\section{Patients}

In total, 75 formalin-fixed paraffin-embedded tumor samples containing a total of 86 germ cell tumor components were obtained from patients who underwent orchiectomy at the Department of Urology, Kyushu University Hospital, Fukuoka between 1990 and 2015. None of the patients had undergone preoperative chemotherapy or radiation therapy. In all the cases, the diagnosis was based on light microscopic examination with hematoxylineosin (HE) staining according to the most recent World Health Organization classification [14]. In addition, immunoperoxidase procedures were performed using the streptavidin-biotin peroxidase or universal immunoperoxidase polymer method (Histofine; Nichirei, Tokyo) when necessary to confirm the diagnosis. Consequently, 58 seminoma (48 pure seminoma and 10 seminoma in mixed tumors), 18 embryonal carcinoma (9 pure embryonal carcinoma and 9 in mixed tumors) and 10 yolk sac tumor (all in mixed tumors) were identified. We excluded choriocarcinoma variants from this study because we had only a single choriocarcinoma case, and we excluded teratoma variants because teratomas show a wide range of histologies and are thus difficult to examine by immunohistochemical staining. In addition, frozen materials were available in 12 cases $(7$ seminomas, 2 embryonal carcinomas, 1 mixed embryonal carcinoma and yolk sac tumor, 1 yolk sac tumor and 1 normal testis) obtained between 2004 and 2009.

This study was carried out in accordance with the principles embodied in the Declaration of Helsinki, and was approved by the Ethics Committee of Kyushu University (IRB No. 27-230).

\section{Cell culture and reagents}

The human embryonal carcinoma cell lines NEC8 and NEC14 were purchased from the Riken Bioresource Center (Tsukuba, Japan) and cultured in RPMI-1640 medium with 10\% fetal bovine serum (FBS). The cells were incubated in a humidified atmosphere of $5 \% \mathrm{CO} 2$ at $37^{\circ} \mathrm{C}$.

\section{Immunohistochemistry}

The immunohistochemical study was performed using the universal immunoperoxidase polymer method (Histofine; Nichirei) [15, 16]. The primary monoclonal antibodies used in this study were anti-MPHOSPH1 (NBP1-88042, dilution 1:100; Novus Biologicals), anti-pSTAT3 (\#9131, dilution 1:100; Cell Signaling Technology, Danvers, MA) and anti-SOCS3 (ab16030, dilution 1:100; Abcam, Cambridge, MA).

The nuclear expressions of MPHOSPH1 and pSTAT3, and cytoplasmic expression of SOCS3 were assessed. For scoring MPHOSPH1 and SOCS3 expression, the proportion of stained cells was divided into 5 categories as follows: $0,0-10 \%$; 1 , $10-25 \% ; 2,25-50 \% ; 3,50-75 \%$; and $4,>75 \%$ of cells were stained positive. Staining intensity was divided into 4 categories as follows: 0, negative; 1, weak; 2, intermediate; and 3, strong staining intensity. Finally, we used the product of the proportion score multiplied by the intensity score for evaluation. High expressions of MPHOSPH1 and SOCS3 were defined as a product score of 9-12. This definition was chosen because, when a cut-off score of 1-7 was used, all the cases of embryonal carcinoma and 9 of 10 cases of yolk sac tumor belonged to the high-MPHOSPH1 expression group. Thus we determined 8 as the appropriate cut-off score. For scoring pSTAT3 expression, the nuclear positivity of pSTAT3 was evaluated in accordance with former reports $[12,13$, 17]. Namely, the proportion of stained cells was divided into 3 categories as follows: $0,0-10 \%$; $10-50 \%$; and $2,>50 \%$ of cells were stained positive $[12$, 17]. Three pathologists (T. A., K. K., F. K.) independently evaluated the immunohistochemical staining for each sample.

\section{Western blot analysis}

Protein was extracted from the frozen samples and transfected cell lines using lysis buffer (PROPREP Protein Extraction Solution; iNtRON Biotechnology, Seongnam, Korea) according to the manufacturer's instructions. The protein concentration was quantified with a protein assay (Bio-Rad Laboratories, Hercules, CA) based on the Bradford method. A similar amount of lysate $(20 \mu \mathrm{g})$ was separated by $12 \%$ 
SDS-PAGE and transferred onto polyvinylidene difluoride microporous membranes (GE Healthcare Bio-Sciences, Piscataway, NJ) using a semidry blotter. The transferred membranes were incubated overnight at $\mathrm{f} 4{ }^{\circ} \mathrm{C}$ with primary antibodies for MPHOSPH1 (1:500; Novus Biologicals, Littleton, NH), pSTAT3 (1:1000; Cell Signaling Technology) and SOCS3 (1:1000, Abcam). The antibody for $\beta$-actin (1:10000; Millipore, Bedford, MA) was used as an internal control. The membranes were then incubated overnight at $4^{\circ} \mathrm{C}$ with secondary antibodies. The bound antibody was visualized using an ECL kit (GE Healthcare Bio-Sciences) and the membranes were exposed to X-ray film (GE Healthcare Bio-Sciences) [18-20]. The protein levels were standardized by actin using an image analyzer (Ez-Capture MG; ATTO, Tokyo), and the expression signal relative to the standardized signal was regarded as the level of expression for each sample.

\section{MPHOSPHI knockdown by small-interfering RNA (siRNA)}

An siRNA targeting MPHOSPH1 (ON-TARGET plus Smart Pool siRNAs-siMPHOSPH1) and a non-targeting siRNA control (ON-TARGET plus nontargeting siRNA) were purchased from Dharmacon (Chicago, IL). Transfection was performed according to the manufacturer's forward-transfection protocol using Lipofectamine RNAiMAX (Life Technologies, Carlsbad, CA). Briefly, cancer cells $\left(5 \times 10^{5}\right)$ were seeded in 6-well plates and incubated for $24 \mathrm{~h}$ to allow adherence. siRNAs (25 pmol) and Lipofectamine (15 ul) were diluted in 250 ul of Opti-MEM (Life Technologies) without serum, then incubated for 5 $\mathrm{min}$ at room temperature. The plates were washed, and $250 \mathrm{ul}$ of the mixture composed of siRNA, Lipofectamine and Opti-MEM which has been prepared before was added to the seeded cells (the final siRNA concentration was 25 pmol for the cells). Then, each well was supplemented with $2 \mathrm{ml}$ of RPMI-1640 with $10 \%$ FBS without antibiotics (for a final volume of 2.25 $\mathrm{ml}$ per well), and the cells were further incubated for 24 or $48 \mathrm{~h}$. Transfected cells were used in the subsequent experiments at $24 \mathrm{~h}$ post-transfection.

\section{Cell migration and invasion assays}

Cell migration assays were conducted with transfected cell lines using uncoated Transwell inserts (BD Biosciences, San Diego, CA).

Cell invasion assays were conducted with transfected cell lines using a 24-well Biocoat Matrigel invasion chamber (BD Biosciences) according to the manufacturer's protocol and the methods described previously [21, 22].

$1 \times 10^{4}$ transfected cells were seeded into the upper chamber in serum-free media. The outer wells were filled with media containing 10\% FBS. The cells were incubated at $37^{\circ} \mathrm{C}$ with $5 \% \quad \mathrm{CO} 2$ for $24 \mathrm{~h}$ (migration assay) or $36 \mathrm{~h}$ (invasion assay), and then nonmigrating or noninvading cells were removed by wiping the chamber surface with a cotton swab. Cells that had migrated or invaded through the filter and adhered to its lower surface were fixed and stained with hematoxylin and eosin (H\&E). The number of migrating or invading cells on the membrane was counted in five microscopic fields $(\times 400)$. The results are expressed as the mean number of cells per field. Each assay was conducted in quadruplicate and repeated three times.

\section{Cell proliferation assay}

Transfected cells were seeded in 96-well plates at a concentration of 5000 cells/ well in serum-containing growth medium and incubated for $24 \mathrm{~h}$ and $48 \mathrm{~h}$. Cell viability was evaluated by using a Cell Counting Kit 8 (CCK-8; Dojindo Molecular Technologies, Kumamoto, Japan) according to the manufacturer's instructions and previous studies $[23,24]$. The absorbance at 450 $\mathrm{nm}$ was measured by a microplate reader (Model 680 Microplate Reader; Bio-Rad Laboratories). All experiments were done in quintuplicate and repeated three times.

\section{Colony Formation Assays}

Anchorage-independent growth was assessed by colony formation in soft agar as previously described [21]. A bottom layer of $0.35 \%$ agarose/RPMI with $10 \%$ FBS was plated on a 6-well dish. Cells $\left(1.5 \times 10^{4}\right)$ were suspended in $0.3 \%$ agarose/RPMI with $10 \%$ FBS, overlaid on the bottom layer and incubated for 14 days. Colonies were stained with crystal violet $(0.005 \%)$ for $60 \mathrm{~min}$ and counted under a light microscope.

\section{Statistical analysis}

We used the Fisher's exact test and Mann-Whitney $U$ test as appropriate to evaluate associations between two variables. Two-sided $P$-values less than 0.05 were considered to indicate statistical significance. The data analyses were conducted with the JMP statistical software package (version 12.0.2; SAS Institute, Cary, NC).

\section{Results}

\section{Patient characteristics}

The clinical and pathological characteristics of the 75 patients with testicular germ cell tumor are summarized in Table 1 . The patients ranged in age from 21 to 65 years old. A total of 86 histological components, including 58 seminoma (48 pure 
seminoma and 10 seminoma in mixed tumors), 18 embryonal carcinoma ( 9 pure embryonal carcinoma and 9 in mixed tumors) and 10 yolk sac tumor (all in mixed tumors) were detected. Survival data were available in all cases. Only 2 patients died, 1 with pure seminoma and 1 with mixed tumor (embryonal carcinoma and yolk sac tumor); the remaining 73 patients survived without recurrence.

\section{Immunohistochemical result}

Table 2 summarizes the immunostaining results. Representative histopathological images of seminoma, embryonal carcinoma and yolk sac tumor components are shown in Figure 1. The embryonal carcinoma and yolk sac tumor components exhibited a higher rate of MPHOSPH1 expression compared with the seminoma component (16/18 (89\%), 8/10 $(80 \%)$ and $5 / 58 \quad(9 \%)$ specimens exhibited high-MPHOSPH1 expression for the embryonal carcinoma, yolk sac tumor, and seminoma components, respectively). Statistically significant differences in MPHOSPH1 expression were recognized between embryonal carcinoma and seminoma, and between yolk sac tumor and seminoma $(p<0.001$ for both). Statistically significant differences were also observed in pSTAT3 expression between seminoma and yolk sac tumor (22/58 specimens ( $38 \%)$ vs. $8 / 10$ specimens $(80 \%) ; p=0.016)$ and in high-SOCS3 expression between seminoma and embryonal carcinoma $(15 / 58$ specimens $(26 \%)$ vs. $11 / 18$ specimens $(61 \%) ; p=0.002)$.

We next examined the relationship between MPHOSPH1 expression and clinicopathological variables, pSTAT3 expression, and SOCS3 expression. In total, MPHOSPH1 was significantly associated with younger age, high pSTAT3 expression, high SOCS3 expression and distant metastasis $(p=0.02$, $p=0.01, p=0.007, p=0.001$, respectively) (Table 3). No significant relations were identified between any of the clinical and pathological variables (pathological $\mathrm{T}$ stage, lymph node status, elevated tumor markers (any of LDH, lactate dehydrogenase; AFP, alphafetoprotein; HCG, human chorionic gonadotropin), lymphatic vessel permeation and vascular permeation) and MPHOSPH1 expression.

We carried out an additional analysis for each histological component (Supplementary Table S4AC). In seminoma, a significant correlation was noted between MPHOSPH1 and pSTAT3 $(p=0.04)$ (Supplementary Table S4A). There were no significant correlations between MPHOSPH1 and the other parameters. In embryonal carcinoma and yolk sac tumor, no significant association was revealed between MPHOSPH1 and any of the clinicopathological parameters (Supplementary Table S4B and S4C).
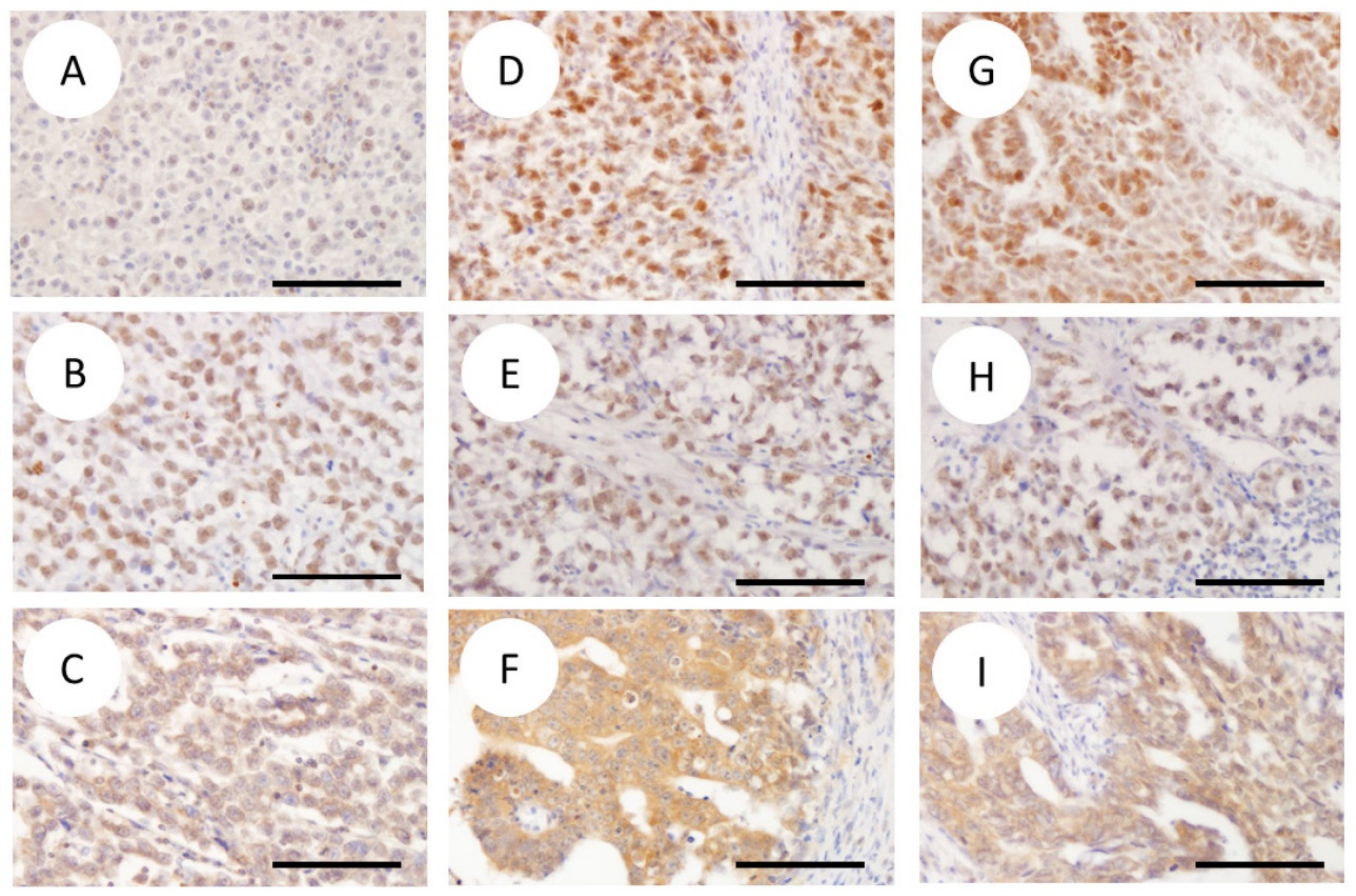

Figure 1. Representative immunohistochemical images of MPHOSPHI (A, D, G), pSTAT3 (B, E, H) and SOCS3 (C, F, I) (original magnification $\times 200)$. The columns represent seminoma (SE) (A-C), embryonal carcinoma (EC) (D-F) and yolk sac tumor (YST) (G-I). Note the strong nuclear expression of MPHOSPH1 in EC (D) and YST (G) compared with that of SE (A). Stronger cytoplasmic expression of SOCS3 is also observed in EC (F) than in SE (C) and YST (I). EC (D) and YST (G) showed immunoreactivity for MPHOSPHI, an evaluated proportion score of 4 (= >75\%), an intensity score of 3 (= strong), and a total score of 12 . In SE (A), the proportion score was 2 (= 25-50\%), the intensity score was 2 (= intermediate), and the total score was 4. Diffuse immunoreactivity for pSTAT3 evaluated as a score of 2 was observed (B, E, H). SE (C) and YST (I) showed immunoreactivity for SOCS3, with an evaluated proportion score of 4 (=>75\%), an intensity score of 2 (= intermediate), and a total score of 8 . In EC (F), the proportion score was 4 (= >75\%), the intensity score was 3 (= strong), and the total score was 12 . Scale bars indicate 20 um. Abbreviations: SE, seminoma; EC, embryonal carcinoma; YST, yolk sac tumor. 
Table 1. Clinical and pathological findings of 75 TGCT patients

\begin{tabular}{|c|c|c|}
\hline Parameter & $\mathrm{N}=75$ & Percentage \\
\hline \multicolumn{3}{|l|}{ Age (year) } \\
\hline median (range) & $34(21-65)$ & \\
\hline \multicolumn{3}{|l|}{ Histology } \\
\hline Pure SE & 48 & $64 \%$ \\
\hline $\mathrm{SE}+\mathrm{EC}$ & 3 & $4 \%$ \\
\hline $\mathrm{SE}+\mathrm{YS}$ & 2 & $3 \%$ \\
\hline $\mathrm{SE}+\mathrm{YS}+\mathrm{CC}$ & 1 & $2 \%$ \\
\hline SE + Teratoma & 4 & $5 \%$ \\
\hline Pure EC & 9 & $12 \%$ \\
\hline$E C+Y S$ & 5 & $7 \%$ \\
\hline $\mathrm{EC}+$ Teratoma & 1 & $1 \%$ \\
\hline YS + Teratoma & 2 & $3 \%$ \\
\hline \multicolumn{3}{|c|}{ Pathological T stage } \\
\hline pT1 & 39 & $52 \%$ \\
\hline pT2 & 27 & $36 \%$ \\
\hline pT3 and pT4 & 9 & $12 \%$ \\
\hline \multicolumn{3}{|l|}{ LN metastases } \\
\hline Present & 24 & $32 \%$ \\
\hline Absent & 51 & $68 \%$ \\
\hline \multicolumn{3}{|c|}{ Distant metastasis } \\
\hline Present & 14 & $19 \%$ \\
\hline Absent & 61 & $81 \%$ \\
\hline \multicolumn{3}{|c|}{ Tumor marker (any of LDH, AFP, HCG) } \\
\hline Elevated & 49 & $65 \%$ \\
\hline Normal & 26 & $35 \%$ \\
\hline \multicolumn{3}{|c|}{ Vascular permeation } \\
\hline Present & 31 & $41 \%$ \\
\hline Absent & 44 & $59 \%$ \\
\hline \multicolumn{3}{|c|}{ Lymphatic permeation } \\
\hline Present & 21 & $28 \%$ \\
\hline Absent & 54 & $72 \%$ \\
\hline
\end{tabular}

Abbreviations: SE, seminoma; EC, embryonal carcinoma; YST, yolk sac tumor; $\mathrm{CC}$, choriocarcinoma

\section{MPHOSPHI expression analysis in frozen samples}

Next, we conducted a protein analysis in 12 frozen samples by Western blotting (Fig. 2). Compared with normal testicular tissue, all cases of TGCTs showed high expression of MPHOSPH1. Moreover, the embryonal carcinoma and yolk sac tumor samples expressed higher levels of MPHOSPH1 than seminoma. We then compared the immunohistochemistry score and the protein level standardized by that of actin. These values were almost concordant with each other, and thus the immunohistochemical evaluations of MPHOSPH1, pSTAT3 and SOCS3 were shown to be reliable.

\section{MPHOSPH1 knockdown by small-interfering RNA (siRNA)}

We confirmed the effectiveness of MPHOSPH1 siRNA by Western blotting (Fig. 3A). We also examined the levels of pSTAT3 and SOCS3 in embryonal carcinoma cell lines. The expression of MPHOSPH1 was decreased in the MPHOSPH1 siRNA-transfected cells compared to the controls in embryonal carcinoma cell lines. However, the expression levels of pSTAT3 and SOCS3 were rarely altered by MPHOSPH1 knockdown as assessed by immunoblot analysis.

\section{Antimigration, anti-invasion, antiproliferation and anti-colony formation effects of MPHOSPHI knockdown in embryonal carcinoma cell lines}

Both of the siMPHOSPH1-transfected embryonal carcinoma cell lines, NEC8 and NEC14, showed reduced cell migration and invasion compared to the control siRNA-transfected cells $(p<0.001$ for both) (Fig. 3B, 3C). Similarly, reduced cell proliferation and colony formation were also recognized in NEC8 and NEC14 ( $p<0.001$ for both) (Fig. 3D, 3E).

Table 2. Immunostainning result of 86 TGCT components

\begin{tabular}{|c|c|c|c|}
\hline \multicolumn{4}{|l|}{ MPHOSPH1 } \\
\hline & Score $0-8(n=57)$ & Score 9-12 $(n=29)$ & $p$ value \\
\hline Seminoma & $53(91 \%)$ & $5(9 \%)$ & SE-EC: $p<0.001$ \\
\hline $\begin{array}{l}\text { Embryonal } \\
\text { carcinoma }\end{array}$ & $2(11 \%)$ & $16(89 \%)$ & SE-YS: $p<0.001$ \\
\hline Yolk sac tumor & $2(20 \%)$ & $8(80 \%)$ & EC-YS: $p=0.374$ \\
\hline \multicolumn{4}{|l|}{ pSTAT3 } \\
\hline & Score $0(n=46)$ & Score $\geqq 1(n=40)$ & $p$ value \\
\hline Seminoma & $36(62 \%)$ & $22(38 \%)$ & SE-EC: $p=0.255$ \\
\hline $\begin{array}{l}\text { Embryonal } \\
\text { carcinoma }\end{array}$ & $8(44 \%)$ & $10(56 \%)$ & SE-YS: $p=0.016$ \\
\hline Yolk sac tumor & $2(20 \%)$ & $8(80 \%)$ & EC-YS: $p=0.205$ \\
\hline \multicolumn{4}{|l|}{ SOCS3 } \\
\hline & Score $0-8(n=57)$ & Score 9-12 $(n=29)$ & $p$ value \\
\hline Seminoma & $43(74 \%)$ & $15(26 \%)$ & SE-EC: $p=0.002$ \\
\hline $\begin{array}{l}\text { Embryonal } \\
\text { carcinoma }\end{array}$ & $7(39 \%)$ & $11(61 \%)$ & SE-YS: $p=0.267$ \\
\hline Yolk sac tumor & $7(70 \%)$ & $3(30 \%)$ & EC-YS: $p=0.176$ \\
\hline
\end{tabular}

Table 3. MPHOSPHI expression and clinicopathological variables

\begin{tabular}{|c|c|c|c|c|}
\hline \multirow[t]{2}{*}{ Germ cell tumor total } & \multicolumn{3}{|c|}{ MPHOSPH1 expression } & \multirow[b]{2}{*}{$P$ value } \\
\hline & total & High $(n=29)$ & Low $(n=57)$ & \\
\hline Age & & $31.8(21-56)$ & $37.6(21-65)$ & 0.02 \\
\hline$\geqq \mathrm{pT} 2$ & 41 & 16 & 25 & 0.32 \\
\hline$\geqq \mathrm{N} 1$ & 26 & 9 & 17 & 0.91 \\
\hline$\geqq \mathrm{M} 1$ & 16 & $11(38 \%)$ & $5(9 \%)$ & 0.001 \\
\hline $\begin{array}{l}\text { Tumor marker elevated } \\
\text { (any of LDH, AFP, HCG) }\end{array}$ & 59 & 23 & 36 & 0.12 \\
\hline lymphatic permeation & 23 & 7 & 16 & 0.7 \\
\hline vascular permeation & 35 & 14 & 21 & 0.32 \\
\hline pSTAT3 high expression & 40 & $19(66 \%)$ & $21(37 \%)$ & 0.01 \\
\hline SOCS3 high expression & 51 & $23(79 \%)$ & $28(49 \%)$ & 0.007 \\
\hline
\end{tabular}




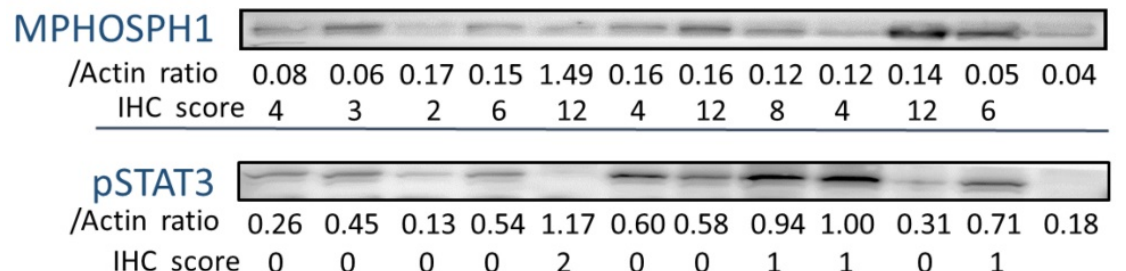

IHC score $00 \begin{array}{llllllllll}0 & 0 & 0 & 2 & 0 & 0 & 1 & 1 & 0 & 1\end{array}$

\section{SOCS3}

/Actin ratio $\begin{array}{llllllllllllll}0.86 & 0.96 & 0.67 & 0.85 & 0.55 & 0.46 & 0.78 & 0.96 & 0.95 & 0.04 & 0.98 & 0.22\end{array}$

$\begin{array}{llllllllllll}\text { IHC score } & 12 & 8 & 6 & 2 & 8 & 0 & 8 & 8 & 8 & 2 & 12\end{array}$

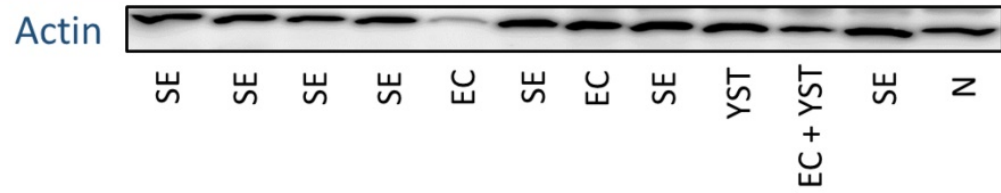

Figure 2. The proteins extracted from 12 frozen samples were subjected to SDS-PAGE and Western blotting. The expression signals of MPHOSPH1, pSTAT3 and SOCS3 were standardized by that of actin and the ratio was taken as the protein level for each sample. The immunohistochemical scores of the same cases are noted. Abbreviations: SE, seminoma; EC, embryonal carcinoma; YST, yolk sac tumor; N, normal testis.

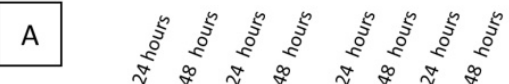

$\mathrm{MPHOSPH}$ $\begin{array}{lllllllllllll}\text { /Actin ratio } & 0.56 & 0.12 & 0.79 & 0.57 & 0.37 & 0.02 & 0.13 & 0.27\end{array}$ PSTAT3

/Actin ratio $0.060 .170 .100 .04 \quad 0.020 .110 .110 .15$

SOCS3

/Actin ratio $3.843 .831 .241 .82 \quad 1.23 \quad 0.390 .82 \quad 2.93$

Actin

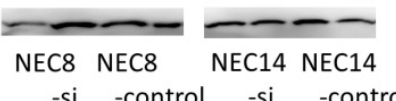

-si -control -si -control

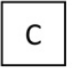

Invasion Assay
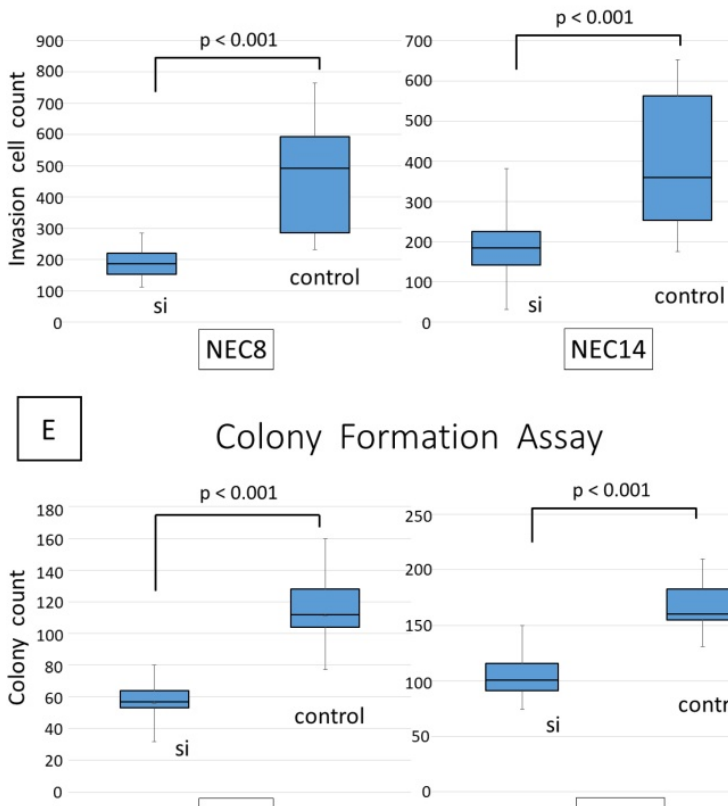

Colony Formation Assay

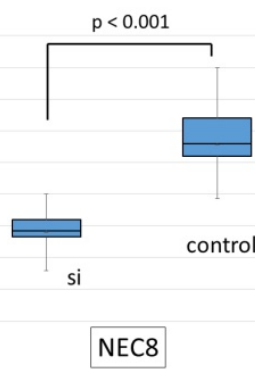

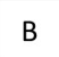

Migration Assay
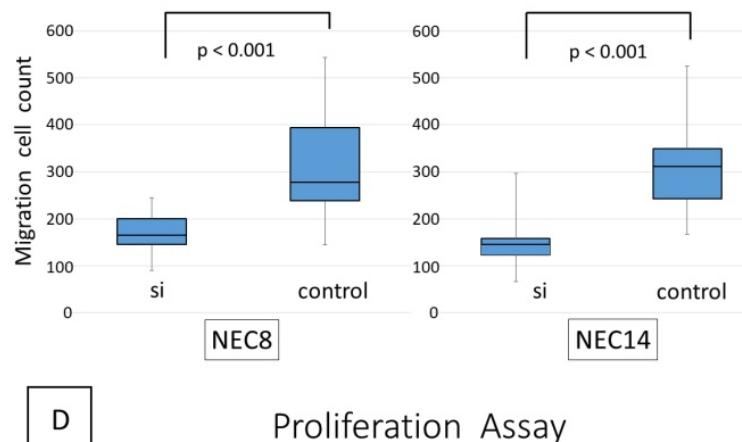

Proliferation Assay
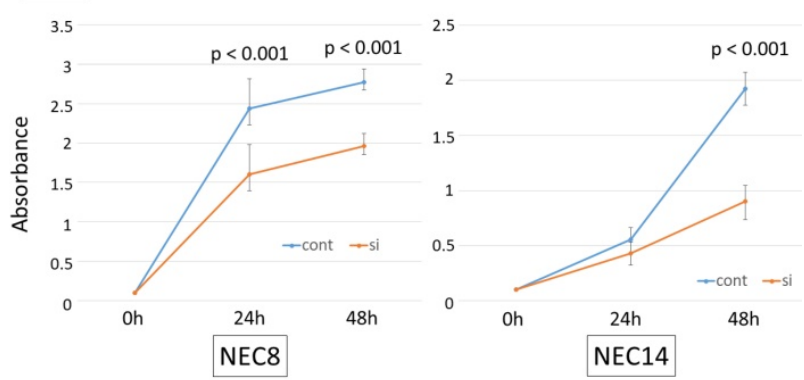

Fig. 3 The efficacy of MPHOSPH1 knockdown using small-interfering RNA (siRNA). A. Protein was extracted from the cell lines at $24 \mathrm{~h}$ and $48 \mathrm{~h}$ post-transfection. Knockdown of MPHOSPH1 was confirmed by Western blotting $(\mathbf{A})$. MPHOSPHI knockdown significantly reduced cell migration $(\mathbf{B})$ and cell invasion $(\mathbf{C})$ in both NEC 8 and NEC14 $(p<0.001$ for both). MPHOSPHI knockdown also significantly inhibited cell proliferation (D) and colony formation (E) in both of the cell lines $(p<0.001$ for both). 


\section{Discussion}

High expression of MPHOSPH1, one of the most promising oncogenic targets, has been observed in various tumors, such as urothelial carcinoma of the urinary bladder and hepatocellular carcinoma $[8,11]$. In a phase I clinical trial of a five-peptide cancer vaccine therapy, one of which was derived from MPHOSPH1, combined with cyclophosphamide for advanced gastrointestinal, lung or uterine cervical cancer, the treatment was well tolerated without any adverse events [25]. In the same trial, tumor-associated antigen-specific $\mathrm{T}$ cell responses were simultaneously induced and significantly associated with longer overall survival. A phase I/II study of cancer peptide vaccine S-288310 including the MPHOSPH1 oncoantigen in patients with advanced urothelial carcinoma of the urinary bladder showed sufficient tolerance and effective induction of peptide-specific cytotoxic T lymphocytes, which were correlated with longer survival for the patients [26].

In the present study, the group of patients with non-seminoma TGCTs - i.e., patients with embryonal carcinoma or yolk sac tumor-showed high immunoexpression for MPHOSPH1 (89\% and 80\%, respectively). In contrast, high immunoexpression for MPHOSPH1 was infrequent (9\%) in the patients with seminoma. Significant differences in MPHOSPH1 expression were observed between each of these pairs of groups (i.e., embryonal carcinoma vs. seminoma and yolk sac tumor vs. seminoma). According to the National Comprehensive Cancer Network (NCCN), non-seminoma patients show worse prognosis compared with pure seminoma patients [3]. Prognostic information has also been provided by the International Germ Cell Cancer Collaborative Group (IGCCCG) as follows [27]. The 5-year survival rate of patients with TGCT in IGCCCG's poor risk group approximates $48 \%$; however, there is no poor-risk group in seminoma. Overexpression of MPHOSPH1 in the non-seminoma group might indicate that MPHOSPH1 is associated with worse prognosis in TGCTs.

In addition, our study revealed that high expression of MPHOSPH1 interacted with distant metastasis. According to the AJCC/UICC, patients with distant metastasis are categorized in an advanced stage of at least stage III [28]. Hence the relation between MPHOSPH1 and distant metastasis could also indicate that MPHOSPH1 might be associated with worse prognosis.

Therefore, testicular germ cell tumors with MPHOSPH1 overexpression, particularly in the non-seminoma group or patients with distant metastasis, which indicate worse prognosis, may be a new candidate for MPHOSPH1 target therapy.

Previous in vitro studies demonstrated that knockdown of MPHOSPH1 results in the death of human bladder cancer cells and reduced proliferation of human hepatocellular carcinoma cells [8, 11]. Our MPHOSPH1-knockdown embryonal carcinoma cells (NEC8 and NEC14) provided by transfection of siRNA showed suppressed cell migration and invasion compared to the control siRNA-transfected cells. Proliferation and colony formation of embryonal carcinoma cells (NEC8 and NEC14) were also significantly decreased by MPHOSPH1 knockdown. Our results thus support the idea that MPHOSPH1 target therapy could be of benefit for embryonal carcinoma patients.

In a previous report, attenuating MPHOSPH1 inhibited hepatocellular carcinoma cell proliferation in a manner of blocking STAT3 phosphorylation, prolonged mitosis and induction of apoptosis [11]. STAT3 has been reported to contribute to tumor progression in some kinds of malignant neoplasms, such as colorectal adenocarcinoma and ovarian carcinoma [29, 30]. Immunoreactivity of pSTAT3, which is activated from of STAT3, was also reported to indicate poor prognosis in their cases. On the other hand, another article reported that suppressor of cytokine signaling 3 (SOCS3) was a potential inhibitor of STAT3 [12]. In soft tissue leiomyosarcoma and undifferentiated pleomorphic sarcoma, STAT3 was inactivated due to the overexpression of SOCS3 [12, 13].

Our present study revealed that high expression of MPHOSPH1 was correlated with high expression of pSTAT3 and SOCS3 in TGCTs. When we examined each of the histological components, high expression of MPHOSPH1 was correlated with the high expression of pSTAT3 in seminoma. Thus MPHOSPH1 may participate in STAT3 phosphorylation and the MPHOSPH1-pSTAT3 pathway is thought to be involved in tumor cell proliferation in TGCTs. The correlation between the high expressions of MPHOSPH1 and SOCS3 suggests the possibility that feedback activation participates in the MPHOSPH1-pSTAT3 pathway.

We were not able to show a significant relation between MPHOSPH1 and pSTAT3 in embryonal carcinoma and yolk sac tumor by the histological analysis of these components. However, in comparison to their levels in seminoma, both MPHOSPH1 and pSTAT3 were highly expressed in yolk sac tumor $(p<0.001, p=0.016)$. In this study, almost all the cases showed high MPHOSPH1 expression in yolk sac tumor (8 of 10 cases). It might be speculated that the MPHOSPH1-pSTAT3 pathway is activated in yolk sac tumors. However, the lack of 
significant relation between MPHOSPH1 and pSTAT3 may also have been due to the small number of subjects.

In embryonal carcinoma, MPHOSPH1 and SOCS3 were expressed at significantly higher levels than in seminoma $(p<0.001, p=0.002)$. Here again, the lack of significance between MPHOSPH1 and pSTAT3 might have been due to the small number of subjects or the activation of SOCS3 as an inhibitor of STAT3. Alternatively, a pathway other than pSTAT3 might have been involved in the tumor cell growth. Consequently, MPHOSPH1 may be a suitable therapeutic target in TGCTs via the MPHOSPH1pSTAT3 pathway. To clarify the correlation among MPHOSPH1, pSTAT3 and SOCS3, further immunohistochemical and biological studies using a larger number of cases and cell lines will be needed.

High expression of MPHOSPH1 interacted with younger age in our study. The correlation between high expression of MPHOSPH1 and younger age was considered to have been caused by the bias - namely, the patients with high expression of MPHOSPH1 included more cases of embryonal carcinoma and yolk sac tumor, and those with low expression of MPHOSPH1 included more cases of seminoma. Embryonal carcinoma and yolk sac tumor usually appear in patients in their 20s to 30s. Seminoma has a later disease onset than either embryonal carcinoma or yolk sac tumor: it generally occurs in men in their 30 s to 40 s but is also seen in men in their 60 s and above [31]. This is why the relation between high expression of MPHOSPH1 and younger age was recognized in this study.

In conclusion, we analyzed MPHOSPH1 expression in TGCTs and verified the effect of MPHOSPH1 inhibition on embryonal carcinoma cell lines. Many TGCTs, particularly in the non-seminoma group or patients with distant metastases, showed high immunoexpression for MPHOSPH1. The knockdown of MPHOSPH1 was shown to inhibit the migration, invasion, proliferation and colony formation of embryonal carcinoma cell lines. Therefore, MPHOSPH1 could be a new therapeutic molecular target or a target of vaccine therapy for TGCTs, especially tumors with high expression of MPHOSPH1, which tend to show a worse prognosis.

\section{Supplementary Material}

Supplementary tables.

http://www.jcancer.org/v09p4440s1.pdf

\section{Acknowledgments}

The authors are grateful to Momoe Itsumi, Miho Ushijima, Eriko Gunshima and Noriko Hakoda (Department of Urology, Kyushu University) for technical assistance. The authors thank KN International (http://www. kninter.com/) for revising the English used in this article.

\section{Competing Interests}

The authors have declared that no competing interest exists.

\section{References}

1. Rajpert-De Meyts E, McGlynn KA, Okamoto K, Jewett MA, Bokemeyer C. Testicular germ cell tumours. Lancet. 2016; 387(10029): 1762-74.

2. Gori S, Porrozzi S, Roila F, Gatta G, De Giorgi U, Marangolo M. Germ cell tumours of the testis. Crit Rev Oncol Hematol. 2005; 53(2): 141-64.

3. Motzer RJ, Agarwal N, Beard C, Bolger GB, Boston B, Carducci MA, et al. NCCN clinical practice guidelines in oncology: testicular cancer. J Natl Compr Canc Netw. 2009; 7(6): 672-93.

4. O'Callaghan A, Mead GM. Testicular carcinoma. Postgrad Med J. 1997; 73(862): 481-6.

5. Hartmann JT, Kanz L, Bokemeyer C.Drugs. Diagnosis and treatment of patients with testicular germ cell cancer. Drugs. 1999; 58(2): 257-81.

6. Abaza A, Soleilhac JM, Westendorf J, Piel M, Crevel I, Roux A, et al. M phase phosphoprotein 1 is a human plus-end-directed kinesin-related protein required for cytokinesis. J Biol Chem. 2003; 278(30): 27844-52.

7. Talapatra SK, Rath O, Clayton E, Tomasi S, Kozielski F. Depsidones from Lichens as Natural Product Inhibitors of M-Phase Phosphoprotein 1, a Human Kinesin Required for Cytokinesis. J Nat Prod. 2016; 79(6): 1576-85.

8. Kanehira M, Katagiri T, Shimo A, Takata R, Shuin T, Miki T, Fujioka T, et al. Oncogenic role of MPHOSPH1, a cancer-testis antigen specific to human bladder cancer. Cancer Res. 2007; 67(7): 3276-85.

9. Obara W, Ohsawa R, Kanehira M, Takata R, Tsunoda T, Yoshida K, et al. Cancer peptide vaccine therapy developed from oncoantigens identified through genome-wide expression profile analysis for bladder cancer. Jpn J Clin Oncol. 2012; 42(7): 591-600.

10. Liu XR, Cai Y, Cao X, Wei RC, Li HL, Zhou XM, et al. A new oncolytic adenoviral vector carrying dual tumour suppressor genes shows potent anti-tumour effect. J Cell Mol Med. 2012; 16(6): 1298-309.

11. Liu X, Zhou Y, Liu X, Peng A, Gong H, Huang L, et al. MPHOSPH1: a potential therapeutic target for hepatocellular carcinoma. Cancer Res. 2014; 74(22): 6623-34.

12. Bekki H, Kohashi K, Yamada Y, Iura K, Ishii T, Maekawa A, et al. Phosphorylation of STAT3 in Undifferentiated Pleomorphic Sarcoma Is Correlated with a Favorable Prognosis. Pathobiology. 2017; 84(3): 161-169.

13. Setsu N, Kohashi K, Endo M, Yamamoto H, Tamiya S, Takahashi Y, et al Phosphorylation of signal transducer and activator of transcription 3 in soft tissue leiomyosarcoma is associated with a better prognosis. Int J Cancer. 2013; 132(1): 109-15.

14. Moch H, Cubilla AL, Humphrey PA, Reuter VE, Ulbright TM. The 2016 WHO Classification of Tumours of the Urinary System and Male Genital Organs-Part A: Renal, Penile, and Testicular Tumours. Eur Urol. 2016; 70(1): 93-105.

15. Yamashita K, Kohashi K, Yamada Y, Nishida Y, Urakawa H, Oda Y, et al. Primary extraskeletal osteosarcoma: a clinicopathological study of 18 cases focusing on MDM2 amplification status. Hum Pathol. 2017; 63: 63-69.

16. Ishii T, Kohashi K, Iura K, Maekawa A, Bekki H, Yamada Y, et al. Activation of the Akt-mTOR and MAPK pathways in dedifferentiated liposarcomas. Tumour Biol. 2016; 37(4): 4767-76.

17. Dobashi Y, Suzuki S, Sugawara H, Ooi A. Involvement of epidermal growth factor receptor and downstream molecules in bone and soft tissue tumors. Hum Pathol. 2007; 38(6): 914-25.

18. Shiota $M$, Itsumi $M$, Takeuchi A, Imada $K$, Yokomizo A, Kuruma $H$, et al Crosstalk between epithelial-mesenchymal transition and castration resistance mediated by Twist1/AR signaling in prostate cancer. Endocr Relat Cancer. 2015; 22(6): 889-900.

19. Imada K, Shiota M, Kohashi K, Kuroiwa K, Song Y, Sugimoto M, et al. Mutual regulation between Raf/MEK/ERK signaling and Y-box-binding protein-1 promotes prostate cancer progression. Clin Cancer Res. 2013; 19(17): 4638-50.

20. Sugimoto $M$, Kohashi K, Itsumi M, Shiota M, Abe T, Yamada $Y$, et al. Epithelial to Mesenchymal Transition in Clear Cell Renal Cell Carcinoma with Rhabdoid Features. Pathobiology. 2016; 83(6): 277-86.

21. Mizuuchi $Y$, Aishima S, Ohuchida $K$, Shindo $K$, Fujino M, Hattori M, et al. Anterior gradient 2 downregulation in a subset of pancreatic ductal adenocarcinoma is a prognostic factor indicative of epithelial-mesenchymal transition. Lab Invest. 2015; 95(2): 193-206.

22. Kuda M, Kohashi K, Yamada Y, Maekawa A, Kinoshita Y, Nakatsura T, et al. FOXM1 expression in rhabdomyosarcoma: a novel prognostic factor and therapeutic target. Tumour Biol. 2016; 37(4): 5213-23.

23. Ozono K, Ohishi Y, Onishi H, Nakamura K, Motoshita J, Kato M, et al. Brain-derived neurotrophic factor/tropomyosin-related kinase B signaling pathway contributes to the aggressive behavior of lung squamous cell carcinoma. Lab Invest. 2017; 97(11): 1332-1342. 
24. Kawamoto M, Onishi H, Ozono K, Yamasaki A, Imaizumi A, Kamakura S, et al. Tropomyosin-related kinase B mediated signaling contributes to the induction of malignant phenotype of gallbladder cancer. Oncotarget. 2017; 8(22): 36211-36224.

25. Murahashi M, Hijikata Y, Yamada K, Tanaka Y, Kishimoto J, Inoue H, et al. Phase I clinical trial of a five-peptide cancer vaccine combined with cyclophosphamide in advanced solid tumors. Clin Immunol. 2016; 166-167: 48-58.

26. Obara W, Eto M, Mimata H, Kohri K, Mitsuhata N, Miura I, et al. A phase I/II study of cancer peptide vaccine S-288310 in patients with advanced urothelial carcinoma of the bladder. Ann Oncol. 2017; 28(4): 798-803.

27. International Germ Cell Cancer Collaborative Group. International Germ Cell Consensus Classification: a prognostic factor-based staging system for metastatic germ cell cancers. J Clin Oncol. 1997; 15: 594-603.

28. Osunkoya AO, Grignon DJ. Practical issues and pitfalls in staging tumors of the genitourinary tract. Semin Diagn Pathol. 2012; 29(3): 154-66.

29. T Kusaba, T Nakayama, K Yamazumi, Y Yakata, A Yoshizaki, T Nagayasu, et al. Expression of p-STAT3 in human colorectal adenocarcinoma and adenoma; correlation with clinicopathological factors. J Clin Pathol. 2005; 58(8): 833-838.

30. Rosen DG, Mercado-Uribe I, Yang G, Bast RC Jr, Amin HM, Lai R, et al. The role of constitutively active signal transducer and activator of transcription 3 in ovarian tumorigenesis and prognosis. Cancer. 2006; 107(11): 2730-40.

31. Oosterhuis JW, Kersemaekers AM, Jacobsen GK, Timmer A, Steyerberg EW, Molier M, et al. Morphology of testicular parenchyma adjacent to germ cell tumours. An interim report. APMIS. 2003; 111(1): 32-40; discussion 41-2. 